\title{
Evaluación de paleokarsts en las plataformas calcáreas del Albiano Superior en México, Guatemala y Honduras
}

\author{
Paleokarst Evaluation in the Upper Albian Calcareous \\ Platforms in Mexico, Guatemala and Honduras
}

\author{
Carrasco-Velázquez B.E. \\ Facultad de Ingeniería \\ Universidad Nacional Autónoma de México \\ E-mail:bcar@prodigy.net.mx
}

Información del artículo: recibido: mayo de 2008, reevaluado: enero y agosto de 2010, aceptado: septiembre de 2010

\begin{abstract}
Resumen
Se presenta el resultado de una evaluación en México, Guatemala y Honduras de las rocas en las plataformas carbonatadas del Albiano Superior, que fueron expuestas a condiciones subaéreas por un descenso del nivel del mar, propiciando que los fenómenos físicos y químicos, así como las condiciones de temperatura favorecieran la formación de algún tipo de karsticidad. Se emplea una metodología para identificar un paleokarst, por medio de la petrología, fábrica, geometría y estratigrafía de sus brechas. En la actualidad, únicamente la localidad de Dengandho en la plataforma de Actopan reúne los requisitos para afirmar la presencia de un paleokarst. De los tres países hay literatura que menciona la presencia de "karst" en el Albiano Superior en las plataformas de Coahuila, Valles-San Luis Potosí, Faja de Oro, Córdoba, Artesa-Mundo Nuevo, Chiapas, Guatemala y Honduras. Como muchos de estos trabajos fueron hechos antes de que se estableciera una metodología para la identificación de paleokarsts, y algunas descripciones no permiten verificar si realmente se trata de paleokarst en el sentido estricto; se estima que es necesario investigar y detallar en el campo y en la información geológica del subsuelo, donde hay "paleokarsts". Fuera de los tres países, en Texas, en la plataforma de San Marcos hay rocas del Albiano Superior que han confirmado su paleokarstificación aplicando la metodología citada del estudio de las brechas. En el Golfo de México en el "Jordan Knoll" y en el Escarpe de Campeche, hay estudios geofísicos que citan la presencia de una discordancia en Albiano Superior, pero no se tienen evidencias físicas de ellas, ni muestras de roca.
\end{abstract}

\section{Descriptores}

- plataformas calcáreas

- Albiano Superior

- paleokasts

- México

- Guatemala

- Honduras

- evaluación 


\begin{abstract}
This paper presents the evaluation in México, Guatemala and Honduras of theUpper Albian platform carbonate rocks that were exposed to subaerial conditions by the falling of the sea level, exposing the rocks to the physical, chemical and temperature phenomena allowing for some type of karst formation. There is a methodology for the identification of paleokarsts by the petrology, fabrics, geometry and stratigraphy of the breccias. Only at Dengandho in the Actopan Platform there are the evidences to confirm a paleokarst. In the three countries mentioned there is literature related to the presence of "paleokarst" in the Upper Albian rocks of the Coahuila, Valles-San Luis Potosí, Golden Lane, Córdoba, Artesa-Mundo Nuevo, Chiapas, Guatemala and Honduras Platforms. As much of the work was done before the time when the methodology for paleokarsts was stablished, muchof the descriptions do not allow confirmation of the paleokarsts presence. Therefore, it is necessary to carry out field research and to study the subsurface rocks in order to confirm the paleokarst structures. In Texas, at the San Marcos Platform (Upper Albian rocks), the studies of the breccias confirm a paleokarst structure in the rocks. In the deep waters of the Gulf of Mexico the geophysics research done at the Jordan Knoll and the Campeche Scarp has discovered at Upper Albian rocks an unconformity at the Upper Albian rocks, but there are no physical evidences or rocks to confirm a paleokarst.
\end{abstract}

\section{Introducción}

Como antecedentes del estudio de paleokarts se tiene que ya desde la segunda mitad de los siglos XIX y en la primera mitad del XX a nivel global se identificaron la mayoría de las discordancias estratigráficas expresadas en algunos casos por paleokarsts. En México se reconocen las discordancias estratigráficas en las rocas calcáreas de la Sierra Madre Oriental por los estudios geológicos de fines del siglo XIX y de principios del siglo XX, donde se observó la existencia de "une lacune et discordance d'erosion entre le calcaire mésocrétacé et le Crétacé supérieur" ("Una discordancia y hiatus entre la rocas calcáreas del mesocretácico y el Cretácico superior") (Burckhardt, 1930), en la Sierra del Abra, S.L.P., así mismo que "des mouvements orogéniques survenus au debut du Mésocrétace auraient plissé le calcaire du Crétacé inférieur et aprés une émersion de courte durée la mer mésocrétaceserait revenue et ses dépôts auraient recouvert en transgression les calcaires du Crétacé inférieur" ("los movimientos orogénicos que sobrevinieron al inicio del Mesocretácico plegaron las rocas calcáreas del Cretácico inferior y después de una emersión de corta duración del mar mesocretácico, las rocas volvieron a ser cubiertas por una transgresión sobre las rocas calcáreas del Cretácico inferior") (Boese, 1899) en la región de Orizaba; y en un sentido paleogeográfico regional Burckhardt (1930) cita que "dans la région centrale et orientale du pays, aucune discordance n'a été observée á la base du Mésocrétacé" ("en la región central y oriental del país se observan algunas discordancias en la base del Mesocretácico"). Las observaciones de campo hechas por Boese (1899) y Burckhardt (1930), muestran las evidencias de erosión, emersión o discordancias identificadas en las rocas calcáreas.

A principios del Siglo XX, durante la exploración de hidrocarburos en el subsuelo de México es muy citado el hecho de que en la perforación en 1916 del Pozo Cerro Azul núm. 4 (según lo registrado en los reportes de perforación (Viniegra et al., 1970), cuyo objetivo era alcanzar la Formación El Abra, se registró un reventón y el descontrol del pozo que propició que "volaran por el aire junto con los hidrocarburos, fragmentos de estalactitas", estas estalactitas pudieron ser los restos de un paleokarst en la Formación El Abra.

En la geología mexicana, los siguientes profesionistas han aportado algunas evidencias sobre la presencia de posibles "paleokarsts", aunque con diversa terminología: Roehl (1968) ('exposure surfaces, karst flute'), Viniegra et al. (1970) ('limestone caverns'), Carrasco (1971) ('litofacies de erosión karstica'), Coogan et al. (1972) ('subaerial exposure, karst development'), Wilson (1975) ('karstic collapse, cavern formation'), Aguayo (1978) ('episodes of exposure, karst surface'), Colorado et al. (1980) ('tipología de yacimientos paleokarsticos'), Enos et al. (1983) ('karst topography, karst caverns'), Minero (1988) ('karst formation, subaerial exposure surfaces, microkarst, karst event'). 
A la luz de los criterios para la identificación de paleokarsts es conveniente evaluar la información estratigráfica y petrológica de las columnas de roca que han sido datadas como del Albiano Superior en México, Guatemala y Honduras, en las que se han descrito eventos de discontinuidades estratigráficas, ahogamiento de plataformas, microkarsts, rocas paleokársticas, erosión-sepultamiento por sedimentos arcillosos, erosión y vulcanismo contemporáneo, etc.

\section{Metodología de identificación de un paleokarst}

Un paleokarst se puede identificar en afloramientos por medio de la estratigrafía y la petrología de las brechas a nivel macroscópico (Loucks, 1999), además con los estudios de diagénesis, paleontología y geoquímica, es posible obtener el modelo genético y anatómico detallado que dé suficiente información geológica sobre su génesis y evolución.

Los criterios en el estudio de las fábricas de las brechas y la petrología han sido descritos por Lucia (1992), Kerans (1993), Loucks (1999) y Loucks et al. (2001). Para describir las características de los sistemas paleokárticos Loucks (1999) propuso un sistema ternario de clasificación de brechas y depósitos clásticos del relleno en sistemas kársticos. La clasificación exhibe las relaciones entre "brecha de agrietamiento", "brecha caótica" y "sedimentos de relleno de caverna". En la figura 1 se presenta esta clasificación.

La clasificación de las brechas y depósitos clásticos en un sistema kárstico o paleokárstico es simple, pero Loucks (1999), Loucks et al. (2001) y Kerans (1993) su-

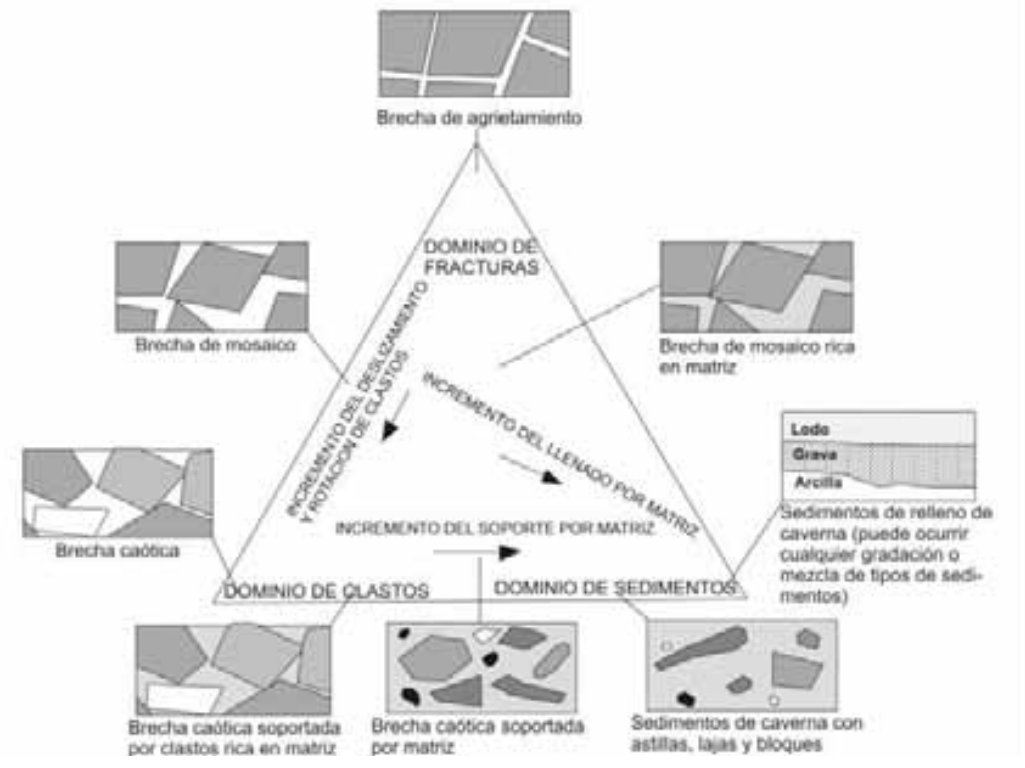

gieren no confundir estas brechas con aquellas de origen tectónico o sedimentario. No hay un orden específico a seguir en esta metodología.

\section{Paleokarst en México}

En México se han encontrado las evidencias petrológicas y estratigráficas en rocas superficiales de la plataforma de Actopan (Carrasco, 1971, 2003; Carrasco et al. 2004), depositadas durante el Albiano Superior en condiciones de perimarea. Estas rocas en un estado avanzado de diagénesis fueron expuestas a condiciones subaéreas por un descenso del nivel del mar, propiciando que la influencia de los fenómenos físicos y químicos, así como las condiciones de temperatura y humedad favorecieran la formación de un karst, que posteriormente fue cubierto por rocas carbonatadas marinas más jóvenes.

En la figura 2 se muestra la localidad de Dengandho, en la plataforma de Actopan, donde Carrasco (2003) detalló la estratigrafía y fábricas petrológicas que conformaron el paleokarst, y en la figura 4 se ilustra la posición de la plataforma de Actopan en extremo sureste de la plataforma de Valles-San Luis Potosí.

En la plataforma de Actopan la Formación El Abra tiene un espesor de 2098 m, en su parte estratigráfica superior se encuentra el paleokarst de Dengandho. En la figura 3 se ilustra su geometría, las rocas encajonantes, la distribución de las brechas y el pasaje vertical de una chimenea del paleokarst.

La sección de Dengandho muestra una exposición del paleokarst de casi $70 \mathrm{~m}$ de anchura y un espesor estratigráfico de $150 \mathrm{~m}$. Está compuesta por cuatro zonas: (A) pilar, (B) brechas, (C) pasaje vertical de una chimenea y (D) cubierta del paleokarst y rocas no karstificadas (equivalentes estratigráficos laterales de las brechas del paleokarst).

Zona A: Consiste principalmente de brechas de agrietamiento y en mosaico. Estas rocas pueden corresponder a una pared de una cueva, o posiblemente a un pilar.

Zona B: Esta zona es la más característica en la sección y está com-

Figura 1. Clasificación de las brechas y depósitos clásticos en un sistema kárstico o paleokárstico (Loucks, 1999). 

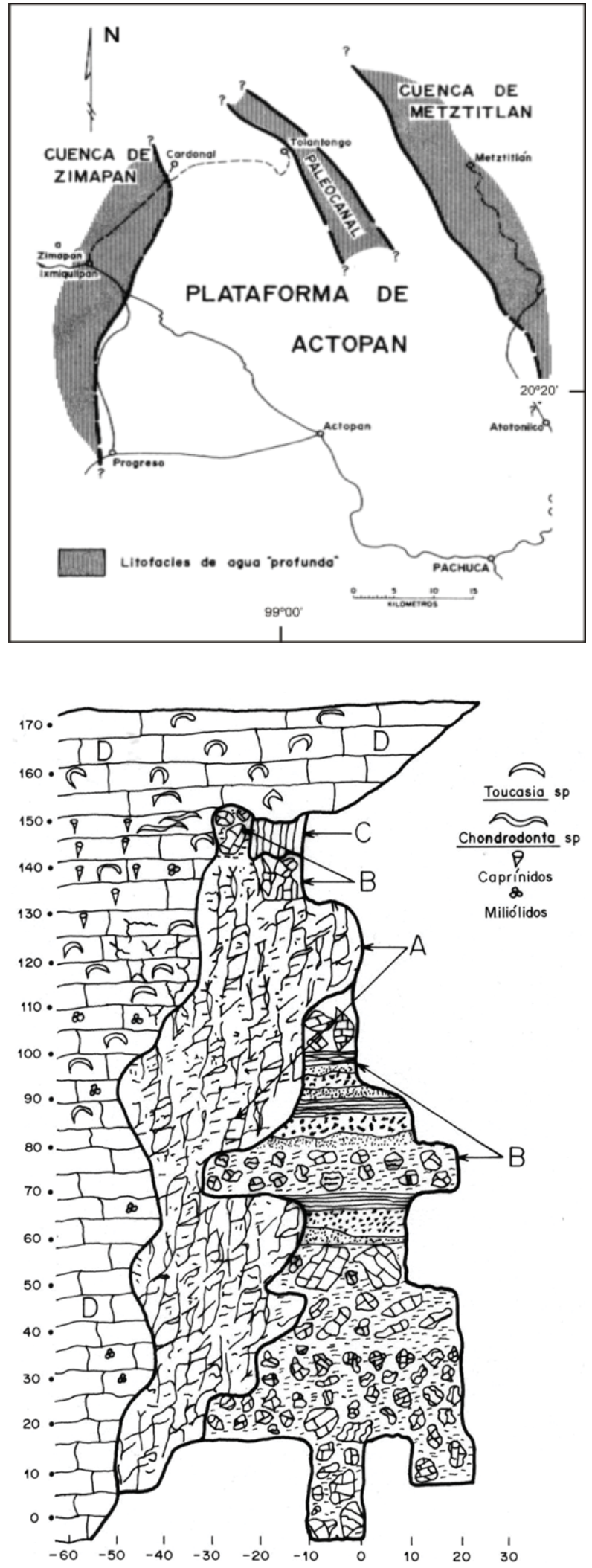

Figura 2. Mapa paleogeográfico de la plataforma de Actopan y localización de la sección de Dengandho, donde la Formación El Abra muestra evidencias del paleokarst. Dengandho se señala con una flecha horizontal de izquierda a derecha
Figura 3. Sección Dengandho del paleokarst de la plataforma de Actopan dibujada en un boceto 'barroco' con escalas horizontal y vertical, ilustrando: (A) pilar, (B) brechas y relleno del paleokarst, (C) pasaje vertical de una chimenea del paleokarst, y (D) rocas no karstificadas equivalentes lateral y estratigráficamente a las paleokarstificadas, junto con la cubierta del paleokarst en la parte superior 
puesta por seis tipos de brechas descritos por Loucks (1999): (1) brecha caótica; (2) brecha caótica soportada por clastos rica en matriz; (3) brecha caótica soportada por matriz; (4) sedimentos de caverna con astillas, lajas y bloques; (5) sedimentos de relleno de caverna y (6) brecha de mosaico con matriz abundante.

Zona C: Su geometría y posición estratigráfica sugieren que esta zona es parte del pasaje vertical de una chimenea.

Zona D: Corresponde a $170 \mathrm{~m}$ de facies no afectadas por el paleokarst (con Toucasia sp., miliólidos, caprinidos del Albiano Superior y Chondrodonta sp.) y son equivalentes estratigráficamente a las rocas afectadas por el paleokarst. Incluye la parte superior de la columna (arriba de $150 \mathrm{~m}$ ) y consiste de $20 \mathrm{~m}$ de capas de wackestone de Toucasia sp. que cubre a los sedimentos del paleokarst.

Este evento podría correlacionarse con otros similares en México, Guatemala y Honduras (figuras 4 y 5), pero faltan estudios detallados petrológicos y de campo para asegurar que los vestigios descritos como "paleokarsts", realmente lo son en un sentido estricto (plataformas de Coahuila, Faja de Oro, Córdoba, Artesa-Mundo Nuevo, Chiapas, Guatemala y Honduras). Quizás esta una labor futura de investigación para obtener detalles.

\section{Otros eventos del Albiano Superior fuera de México}

En Texas, hay estudios sistemáticos del estudio de un paleokarst desde la década de los 60', (un evento contemporáneo al de la plataforma de Actopan) en rocas carbonatadas del Albiano Superior-Cenomaniano del subsuelo de la plataforma de San Marcos (figuras 4 y 5). Donde el desarrollo del paleokarst dio lugar a la formación del acuífero Edwards en el estado de Texas, que es importante para México, ya que las formaciones equivalentes al Grupo Edwards se internan en México y afloran en el estado de Coahuila, en la Serranía del Burro.

La investigación sísmica y de exploración del petróleo ha permitido acumular una gran cantidad de información que aplicada a la estratigrafía sísmica, geología estructural y tectónica han facilitado el entendimiento geológico de estructuras como el "Jordan Knoll" y el Escarpe de la Plataforma de Campeche (Sureste del Golfo de México); según Schlager (1991) en la geología histórica del Cretácico en el Golfo de México, existe la evidencia geofísica de una discordancia en la porción sureste del golfo (Noroeste de Cuba, Sur de Florida y Este de la plataforma de Campeche). En el "Jordan Knoll" (figuras 4 y 5), que es una unidad cretácica se presentan sísmicamente los contornos de una discordancia de carbonatos de periplataforma de edad Albiano-Cenomaniano Inferior. Sumado a lo anterior, Schlager (1991) basándose en información sísmica proporcionada por R.T. Buffler (investigador del Instituto de Geofísica de la Universidad de Texas) considera que en el escarpe del Banco Calcáreo de Campeche (figura 4 y 5) también hay una discordancia del

Figura. 4. Mapa de localización de las plataformas calcáreas del Albiano Superior-Cenomaniano en la provincia geológica del Golfo de México, modificado con datos de Hovorka et al. 1996, Schlager (1991), Eguiluz (1991), Coogan et al. (1972), Enos (1974), Carrasco (2003), Martínez et al. (2001), Ferket et al. (2003), Aguayo et al. (1985), Varela et al. (1997), Williams-R.(2001), Steele (1985), Wilson (1974) y Burkart et al. (1973) 


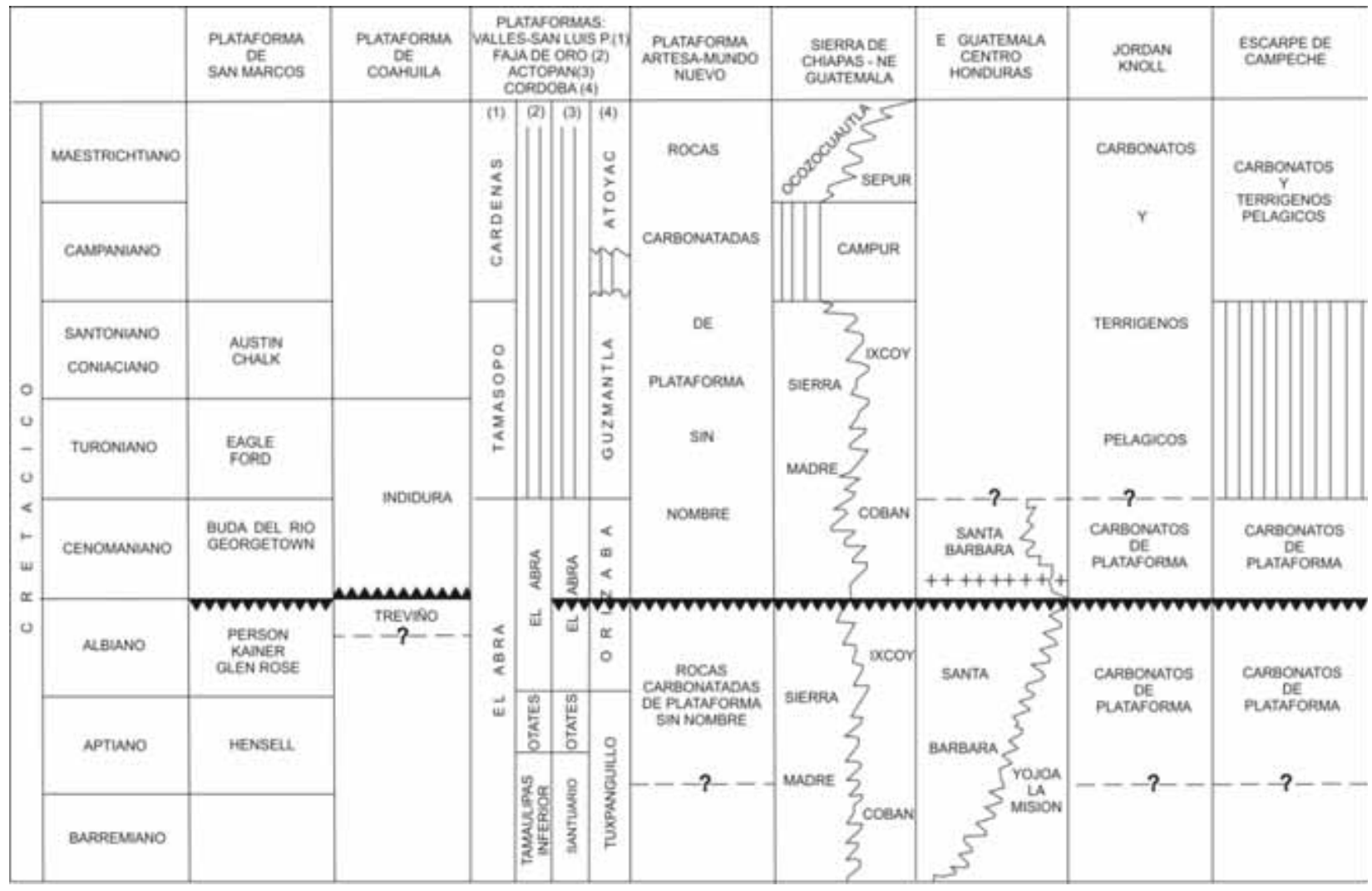

Figura 5. Correlación estratigráfica de las rocas cretácicas en la provincia geológica del Golfo de México, con datos de: Hovorka et al. 1996, Schlager (1991), Eguiluz (1991), Coogan et al. (1972), Enos et al. (1983), Carrasco (2003), Martínez et al. (2001), Aguayo et al. (1985), Varela et al. (1997), Williams-R. (2001), Steele (1985), Wilson (1974) y Burkart et al. (1973).

'Cretácico medio', que es el producto del ahogamiento de la plataforma, por su hundimiento y cobertura en el escarpe, por sedimentos calcáreos pelágicos del Cretácico superior.

La información proporcionada por la geofísica en el "Jordan Knoll" y el Escarpe de la Plataforma de Campeche pueden ser del Albiano Superior, pero de ninguna manera representan pruebas de paleokarsticidad, pero sí del ahogamiento de la plataforma como lo propone Schlager (1991).

\section{Evaluación de plataformas donde pueden existir Paleokarsts}

Plataforma de Coahuila (figura 4):

En el norte de México, Eguiluz (1991) en un estudio localizado en el límite de los estados de Chihuahua, Durango y Coahuila, indica que la cima de la Formación Treviño estuvo sujeta a exposición subaérea y que la Formación Indidura le sobreyace con discordancia pa- ralela, describe que el hiato es del Cenomaniano Inferior (figura 5) y se correlaciona con 'abatimiento eustático global’. Para Eguiluz la Formación Treviño es del Albiano Superior.

Eguiluz (1991), describe el detalle petrológico de las evidencias físicas de la discordancia en las siguientes localidades, es conveniente considerar que las nueve secciones ilustradas en el texto de Eguiluz (figuras 3 a 9), no son secciones detalladas, sólo contienen la litoestragrafía a gran escala, en cambio, sus descripciones petrológicas son adecuadas.

a) Sierra de las Noas, Coah., Eguiluz (1991) cita: “Las evidencias de esta discordancia consisten en que la cima de la Formación Treviño está constituida por caliza packstone de fragmentos de conchas de toucasia y rudistas, que forman una calcarenita de 10 a $20 \mathrm{~cm}$ de espesor, el plano de la superficie de esta última capa tiene irregularidades con oquedades y desgaste por abrasión. A esa superficie le sobreyace un conglomerado que está formado por fragmentos 
de conchas retrabajadas, derivadas de la caliza subyaciente, incluidos en una matriz calcáreo-arcillosa, el conglomerado tiene fragmentos bien redondeados a subredondeados, que varían desde $0.5 \mathrm{~cm}$ hasta $2 \mathrm{~cm}$ de diámetro, su coloración tiene un tinte rojizo provocado por oxidación, pero se desconoce si esta coloración es penecontemporánea o posterior al depósito". No se presentan evidencias o detalles de las brechas, como para suponer un paleokarst.

b) Sierra de Bermejillo, Dgo., Eguiluz (1991) cita: “en la proximidad de una mina situada en el flanco suroeste y parte media de esta sierra; la Formación Treviño está constituida por caliza wackestone de bioclastos bentónicos, su cima está dolomitizada, tiene fantasmas de granos y su contacto superior es una superficie irregular; sobre ésta, descansa una calcarenita de grano grueso con algunos clastos de dolomía negra con sólo unos centímetros de espesor y que contiene numerosos nódulos ferruginosos que le imprimen una coloración ocre, sobre las capas de caliza se aprecian parches de una costra de 1 a $2 \mathrm{~cm}$ de espesor, similar al caliche". Tampoco se presentan evidencias o detalles de las brechas, como para suponer un paleokarst.

c) Sierra del Toro, Chih., Eguiluz (1991) cita: “En el buzamiento sureste de esta sierra, hay un afloramiento que muestra a la Caliza Treviño subyaciendo a la Formación Indidura, en contacto brusco; en el límite interformacional existe una arenisca conglomerática de color ocre o verdoso que está formada por clastos de cuarzo y calcarenitas de grano medio o grueso, embebidos en una matriz arcillosa y cemento calcáreo, con no más de $20 \mathrm{~cm}$ de espesor. En el contacto estratigráfico que aparenta ser regular y paralelo no se encontró fauna diagnóstica". Asimismo no se presentan evidencias o detalles de las brechas, como para suponer un paleokarst.

d) Sierras de Texas y La Peña, Coah., Eguiluz (1991) cita: "la Formación Treviño, constituida por calizas con miliólidos, fragmentos de rudistas y dolomitizada, subyace a un par de centímetros de calcarenita gris clara, formada por clastos retrabajados de la caliza subyacente; éstos varían de grano grueso a medio, con coloración amarillenta por oxidación, embebidos en una matriz arcillo-calcárea sin microfauna determinativa", en otros párrafos el autor menciona que "la unidad Baicuco de (Garza, 1973), con faunas del Cenomaniano Inferior, que sobreyace transicional y concordantemente sobre la Caliza
Treviño, pero subyace discordante a la Formación Indidura, marcada esta irregularidad por una capa de calcarenita, sobre un plano oxidado, con estructuras de corte y relleno, que se toman como indicativos de discordancia". Tampoco en este par de párrafos se presentan evidencias o detalles de las brechas, como para suponer un paleokarst.

e) Sierra de Banderas, Dgo., Eguiluz (1991) cita: "la cima de la Formación Treviño, constituida por calizas wackestone de bentónicos, en facies lagunar, con capas gruesas que pasan en transición a capas delgadas, con mezcla de faunas de organismos bentónicos asociados a Heterohelix sp. y Hedbergella sp.; la cima tiene caliza recristalizada, dolomitizada, arenosa y con estratificación cruzada, fracturas selladas por calcita y abundantes óxidos. En el contacto superior de las calizas aparece un plano que se toma de discordancia, como una superficie irregular con oxidación, que corta a la caliza subyacente, sobre este plano aparecen uno o dos metros de lutita con abundantes óxidos de hierro y posibles foraminíferos". No se presentan evidencias o detalles de las brechas, como para suponer un paleokarst.

f) Pozos Cevallos 1 y Durango 1, quizás la información petrológica de estos dos pozos sea muy importante, Eguiluz únicamente cita: "En el contacto litológico entre las formaciones Treviño e Indidura, en los pozos Cevallos 1 (2,410 m) y Durango 1 (3,287 m), aparecen calcarenitas derivadas de la litología Treviño subyaciente. En ambos pozos, a pocos metros debajo de este contacto, se perdió la recuperación de muestras por existir cavernosidad. El contacto interformacional se infiere discordante como se observó en las secciones de superficie". No hay evidencias o detalles de las brechas, como para suponer un paleokarst.

g) Sierra del Rosario, Dgo., "Esta sierra se ubica paleogeográficamente en el talud sureste de la Plataforma de Coahuila, al noreste de esta sierra se encuentra la Bufa de Mapimí, en donde afloran las facies periarrecifales, mientras que en la Sierra del Rosario, esas facies se interdigitan con la Formación Cuesta del Cura; esta última contiene capas delgadas de caliza mudstone y wackestone de foraminíferos planctónicos, microlaminadas con estructura boudinage, interdigitadas con capas gruesas de la misma litología. El contacto con la Formación Indidura es una superficie irregular, las calizas inferiores al plano de discordancia están muy dolomitizadas y tiene fluorita que 
rellena oquedades y huecos de disolución que ha dado lugar a bonanzas mineralizadas".

Eguiluz (1991) considera que el contacto entre las Formaciones Indidura, Cuesta del Cura-Treviño puede interpretarse desde dos puntos de vista, uno situado en la cuenca y el otro sobre la Plataforma de Coahuila. En el primer caso considera que las brechas conglomeráticas embebidas entre el lodo calcáreo, con microfauna pelágica se formaron en una ambiente acuoso, de plataforma abierta; las características litológicas de los exoclastos indican que provienen de ambientes someros, sujetos a erosión y oxidación; el arreglo caótico o burdamente gradacional, con estructuras de corte como se observa en la Sierra de la Cal, e intercalados entre calizas pelágicas, hacen suponer que los exoclastos fueron arrastrados de una o más plataformas y depositados hacia la cuenca por corrientes de turbidez. En el segundo caso interpreta que las areniscas conglomeráticas y calcarenitas situadas en la base de la Formación Indidura, que sobreyacen al plano de discordancia y que se asocian con disolución, relleno de huecos, recristalización, mineralización, dolomitización, etc. que afectan a la Caliza Treviño, son las bases para suponer la exposición subaérea de la plataforma.

La segunda consideración de Eguiluz (1991) es importante, porque son las localidades donde con los estudios detallados se podrían localizar estructuras y fábricas de paleokarst, como él describió: en la Sierra de las Noas, Sierras de Texas y la Peña, y los Pozos Cevallos 1 y Durango 1, la cima de la Formación Treviño está constituida por:

1) Un plano con irregularidades, oquedades y desgaste por abrasión, y que le sobreyace un conglomerado que está formado por fragmentos de conchas retrabajadas, derivadas de la caliza subyaciente, incluidas en una matriz calcáreo-arcillosa, el conglomerado tiene fragmentos bien redondeados a subredondeados con un tinte rojizo provocado por oxidación.

2) Un par de centímetros de calcarenita gris clara, formada por clastos retrabajados de la caliza subyacente; éstos varían de grano grueso a medio, con coloración amarillenta por oxidación.

3) Que de las calcarenitas derivadas de la 'litología Treviño' subyaciente, asociadas con cavernosidad; no se tienen datos precisos del espesor del conglomerado, pero se supone que es de unos cuantos decímetros o metros.

Las evidencias estratigráficas y litológicas de la formación Treviño de la plataforma de Coahuila y las forma- ciones Person y Georgetown de la plataforma de San Marcos en Texas dejan ver que los dos eventos exposición subaérea en ambas plataformas son contemporáneos, una cuestión no muy clara es que en la plataforma de San Marcos se menciona con seguridad la presencia de brechas de colapso que son características en los paleokarsts, en tanto que en la plataforma de Coahuila, sólo se describe la presencia de conglomerados y cavernosidad en los pozos Cevallos 1 y Durango 1, posiblemente puede tratarse también de brechas de colapso, pero no hay evidencias físicas o descripción detallada de su petrología.

\section{Plataforma de la Faja de Oro}

Petróleos Mexicanos ha considerado durante muchos años que la información geológica de esta plataforma es confidencial, razón por la cual no existe acceso a las muestras de los pozos, columnas estratigráficas, localización de pozos, etc., aquí solo se presentan datos publicados.

La Faja de Oro es una plataforma alargada de sedimentos carbonatados someros de la Formación El Abra (Albiano-Cenomaniano) (figuras 4y 5), orientada de noroeste a sureste con una longitud de $145 \mathrm{~km}$ y $65 \mathrm{~km}$ de ancho; el espesor total de la columna de rocas de plataforma alcanza de los $1500 \mathrm{~m}$ (Wilson, 1975) a los $2000 \mathrm{~m}$ (Guzmán, 1967). Según Wilson (1975) la parte superior de la plataforma fue posiblemente expuesta a erosión en forma intermitente del Cenomaniano al Oligoceno; para Wilson la gran masa de rocas carbonatadas del Albiano-Cenomaniano (Cretácico "medio") es cubierta regionalmente por una discordancia preturoniana en toda la región del Golfo de México, por lo que se formó karsticidad, brechas de colapso y cavernas antes de que hubiera el deposito de las rocas del Cretácico Superior.

Coogan et al. (1972) estudiaron las rocas del subsuelo en la Plataforma de la Faja de Oro, correspondientes a $400 \mathrm{~m}$ de núcleos de roca de 14 pozos, 10 en el área de Poza Rica y 4 en la Plataforma de Faja de Oro; atribuyen la alta porosidad y permeabilidad en las rocas de la Formación El Abra a una erosión subaérea (desarrollo de un karst). No presentan ninguna descripción o evidencias petrográficas de los caracteres macroscópicos y microscópicos de las muestras de los cuatro pozos que estudiaron de la plataforma, donde mencionan caracteres paleokársticos. Loucks (1999) considera que la producción de hidrocarburos en la Faja de Oro proviene de un sistema paleokárstico formado por cavernas abiertas, brechas espeleotemas y rellenos de caverna.

Desafortunadamente mucha información sobre la geología y estratigrafía de la Formación El Abra en la Faja de Oro no ha sido publicada, las consideraciones 
anteriores de Loucks son muy acertadas. En una comunicación personal sobre un estudio inédito del Instituto Mexicano del Petróleo (1974) entre J.T. Castro Mora, C. Predrassini y E. Martínez, encontraron durante el estudio de más de cincuenta pozos perforados en la Faja de Oro, que muchos intervalos estratigráficos en la parte media de la Formación El Abra tenían una 'cavernosidad muy notable', la cual se detectó en las muestras de núcleo, en las pérdidas de la circulación de lodo y en los registros eléctricos, además de que en la secciones sísmicas se notaba pérdida de energía en estos intervalos estratigráficos.

Hay mucho por estudiar y comprobar en la Faja de Oro y es probable que exista un paleokarst del Albiano, aparte de la erosión y karsticidad del Cretácico Superior-Oligoceno.

\section{Plataforma de Valles-San Luis Potosí}

Sobre el borde oriental de la plataforma de Valles-San Luis Potosí (figura 4), en la Sierra del Abra situada a 140 $\mathrm{km}$ al oeste de Tampico, y la cual es el área y localidad tipo de la Formación El Abra, Aguayo (1998) encontró que en la sección ( $250 \mathrm{~m}$ ) aflorante del borde arrecifal de la plataforma, las rocas son del Cenomaniano Inferior, la edad está basada en el estudio de rudistas y de algunas amonitas, estos $250 \mathrm{~m}$ son una mínima parte de los $1800 \mathrm{~m}$ de espesor que la formación tiene en el borde oriental de la Plataforma de Valles-San Luis Potosí, por lo cual no se descarta la posibilidad de localizar el paleokarst del Albiano Superior algunas decenas de metros por abajo del nivel estratigráfico aflorante en la Sierra del Abra.

En la Plataforma de Valles-San Luis Potosí hay únicamente dos trabajos que describen la presencia de paleokarst en la Formación El Abra (descrita originalmente como Caliza El Doctor), uno de ellos está relacionado con la minería, en particular con depósitos de fosforita; Quintus (1982) estudió la zona fosforítica de San Francisco, localizada a unos $15 \mathrm{~km}$ al noreste de Zimapán, Hidalgo en rocas de la Formación El Abra; según él la presencia de la fosforita de San Francisco es consecuencia de la disolución de los fósiles por exposición subaérea (paleokarsticidad). El otro trabajo está relacionado con la tectónica del área del mineral El Doctor, del cual hay dos publicaciones del mismo autor, en una de ellas, Carrillo $(1998,2000)$ en referencia al estudio realizado por Quintus (1982) en San Francisco dice: "Se trata de un yacimiento situado en una uvala desarrollada en las facies del borde occidental de la plataforma carbonatada de Valles-San Luis Potosí. El mayor volumen de fosforita es texturalmente arena, producto de la alteración de fosforita negra sumamente porosa y yace en el fondo de la uvala con espesores que varían entre 0.05 y $5 \mathrm{~m}$. No obstante, en la parte más occidental de la porción visible del borde occidental de la plataforma carbonatada, existen concentraciones fosforíticas en bolsas cársticas de 1-3 m de diámetro y 2-3 m de profundidad"; en la otra publicación Carrillo et al. (2001) describen lo siguiente sobre el área minera de San Francisco: "The tops of both the El Doctor and Valles-San Luis Potosí platforms show paleokarst filled with debris from the Soyatal-Mendez Formations (Eckbert Seibertz, personal communication, 1997). In the San Francisco area, these paleokarsts were filled with phosphorite deposits (Quintus, 1982)".

La información de Quintus (1982), y de Carrillo (2000), Carrillo et al. (2001) no es suficiente y detallada como para asegurar que estas rocas 'paleokársticas' de la Formación El Abra puedan corresponder a un evento del Albiano-Cenomaniano; en comunicación personal del Dr. M. Carrillo (2003), él considera que existe la posibilidad de que sea un evento de paleokarsticidad del Albiano Superior, pero mientras no se tengan las evidencias y el detalle de campo no se puede asegurar su existencia.

Es necesario el estudio petrológico a detalle de estas áreas para confirmar la presencia de paleokársts.

Plataforma de Córdoba, centro-oriente de México

La información sobre un paleokarst en la plataforma cretácica de Córdoba (figura 4 y 5) es muy escasa, solamente hay un resumen presentado en una convención de la American Association of Petroleum Geologists de Martínez et al. (2001) sobre la plataforma, donde describen brevemente que existen rocas kársticas en la Caliza Orizaba (Albiano-Cenomaniano) que forman el yacimiento principal del área; los autores no dan ninguna evidencia física o petrológica del paleokarst. Existe la tarea de comprobarlo.

\section{Plataforma de Artesa-Mundo Nuevo}

La investigación de Aguayo et al. (1985) en las rocas cretácicas de los pozos petroleros del área Reforma-Jalpa permitieron comprobar la erosión de las rocas del Albiano, ellos describen que en estas rocas hay planicies de marea en las que: "litologicamente el área está constituida por micritas finamente laminadas de color gris oscuro, intercaladas con estromatolitos de posible origen algáceo, de color café. Las estructuras sedimentarias más sobresalientes son fracturas de desecación formadas durante los periodos breves de exposición subaérea a que estuvieron sometidos los sedimentos calcáreos; así como brechas de colapso". Posteriormente Varela et al. (1997) en la misma área en los límites entre los Estados de Chiapas y Tabasco (figuras 4 y 5), 
describen que en la plataforma Artesa-Mundo Nuevo, durante la evolución tectónica del área hay un evento en el que hubo: "destrucción de la plataforma durante un levantamiento y erosión que produce una discordancia en el Área Iris".

También en la Plataforma Artesa-Mundo Nuevo donde se localizan los campos petroleros Muspac y Catedral, según Williams (2001) la producción de gas y condensado procede de rocas fracturadas; en su estudio describe que las fracturas están relacionadas con fallas y zonas kársticas y brechas de colapso en rocas depositadas en aguas someras durante el Cretácico medio, además describe que la plataforma continuo su desarrollo durante el Cretácico superior (figura2).

La descripción de Aguayo et al. (1985) es muy clara y no se trata de un evento paleokártico. Los conceptos de Varela et al. (1997) y de Williams (2001) son puntuales y carecen de una descripción amplia, sería conveniente que Petróleos Mexicanos revisara la petrología de las rocas en sus pozos que encontraron estas zonas 'karsticas'.

\section{Sierra Madre del Sur, Chiapas}

En la Sierra Madre del Sur (figuras 4 y 5), la Caliza Sierra Madre fue definida por Boese (1905) como un conjunto de rocas formadas en una plataforma somera del 'Cretácico medio' basándose en la presencia de Chondrodonta munsoni; el espesor de la Caliza Sierra Madre se ha estimado en más de los 2000 metros por distintos geólogos; Steele (1985) y Waite (1985) consideran que hay una columna estratigráfica compuesta del orden de $2575 \mathrm{~m}$.

Castro M. et al. (1975) en la porción noroeste de la Sierra Madre del Sur, establecen que en la columna estratigráfica del Istmo de Tehuantepec el 'Cretácico medio' fue completamente erosionado en la sección Dique $\mathrm{Va}$, quedando como remanentes de la erosión, clastos del Albiano-Cenomaniano en la base del Cretácico Superior, según ellos la discordancia es erosional.

El estudio estratigráfico y petrológico de la Caliza Sierra Madre en la Sierra Madre del Sur tiene problema para su interpretación, ya que diversos geólogos y paleontólogos consideraron que esta formación consiste de dos grandes unidades litológicas, una dolomítica basal y otra superior calcárea (Gutiérrez, 1956; Zavala, 1971; García, 1973; Castro M. et al., 1975); además han hecho la observación de que la dolomitización de las rocas carbonatadas destruyó los atributos petrológicos originales, lo que es un obstáculo para interpretar la estratigrafía de esta formación.

Zavala (1971) considera que en el 'Cretácico medio' la caliza Sierra Madre puede ser dividida en dos miem- bros; el Miembro Cantela que tiene de 420 a 900 m de espesor al suroeste de Tuxtla Gutiérrez y que consiste de dolomías interestratificadas ocasionalmente con biomicrita fosilífera de Nummoloculina heimi; y el Miembro Calizas Cintalapa, que consiste de $750 \mathrm{~m}$ de micrita fosilífera y biomicrita, alternadas con bioespatitas y pelespatitas. Los sedimentos del Cretácico Superior cubren en discordancia al 'Cretácico medio'. Los datos anteriores carecen de detalles estratigráficos, petrológicos y paleontológicos.

Steele (1985) y Waite (1985) investigaron conjuntamente en el campo y laboratorio, cada uno en su especialidad, uno sobre la petrología y el otro sobre la paleontología, además aportaron más datos sobre la problemática de la dolomitización, las evaporitas y la edad de las unidades litológicas y paleontológicas.

Steele (1985) divide la Caliza Sierra Madre en 19 unidades litológicas y Waite (1985) en 11 unidades paleontológicas, concluyendo que la base es probablemente del Barremiano, o del Albiano y la cima del Santoniano Medio.

Steele (1985) describe en la base de la Caliza Sierra Madre, la unidad litológica 1 con un espesor de 828 a 895 m de dolomía gris a café amarillento, formada por brechas producto del colapso de evaporitas, estromatolitos algáceos, fracturas de desecación y dolomía laminar fina, ocasionalmente con sombras de intraclastos y gasterópodos; además con estructuras sedimentarias tales como estratificación cruzada, barrenos, laminaciones de algas y laminaciones de dolomita con anhidrita; en la unidad litológica 2, el mismo autor la describe como consistente de $73.9 \mathrm{~m}$ de una alternancia de mudstone calcáreo, dolomía cristalina fina, wackestone de pellets y miliólidos, y un wackestone de rudistas del tipo de los requiénidos. Con la descripción de las unidades litológicas 1 y 2, Steele (op. cit) aclara muchas de las imprecisiones o dudas anteriores acerca de la génesis de las 'dolomias basales' de la Caliza Sierra Madre; a las dos unidades anteriores Waite les da una edad Albiano Inferior-Albiano Superior (figura 2).

Cubriendo a la Unidad litológica 2, Steele (1985) y Waite (1985) propusieron la Unidad 3 (litológica y paleontológica) como un "intervalo no describible", para ellos es un intervalo estratigráfico no medido directamente por causa del intemperismo y la vegetación, situado entre las unidades 2 y 4 , con un espesor estratigráfico estimado, por su posición estratigráfica de $384 \mathrm{~m}$ y la edad paleontológica por su posición con las unidades 2 y 4 . La edad relativa de la 'unidad 3' es del Cenomaniano Inferior-parte media del Cenomaniano Medio. Según Steele (op. cit) en la base de la unidad 
litológica 4 hay probablemente capas (packstone dolomítico intraclástico) movidas o desplazadas de su lugar de origen, por una disolución karstíca antigua muy profunda formada con piezas irregulares de roca.

Al parecer, los trabajos de investigación de Steele (1985) y Waite (1985) son los más completos, pero carecen de los elementos de petrología y petrofábricas para asegurar la existencia de paleokarst, el área es difícil de trabajar por la vegetación y alteración de las rocas quizás en el futuro algo de sus conceptos y descripciones se puedan ampliar con trabajo de campo.

\section{Guatemala}

Blount y Moore (1969) describen que en el Noroeste de Guatemala (figura 1) en el Cuadrángulo de Chiantla (a $50 \mathrm{~km}$ de los límites con México) en la Caliza Ixcoy, que es equivalente a la Caliza Sierra Madre (Wilson, 1974), hay unos conglomerados y brechas, que ellos llaman litoclásticas, formadas por fragmentos de caliza preexistente, contenidos en una matriz de limo calcáreo o con un cementante de calcita espática, su tamaño varía limo a guijarros, son subangulares a subredondeados y la mayoría contienen fragmentos de rudistas y pelets; la fauna y la litología de los litoclastos es similar a la no litoclástica de la Caliza Ixcoy. Los litoclastos se encuentran principalmente en la parte superior de la Formación Ixcoy (figuras 4 y 5), y el mecanismo de su formación pudo ser por cambios periódicos del nivel del mar; estas rocas están cubiertas por las lutitas y limolitas de las formaciónes Campur-Sepur del Cretácico Superior.

En la región del Departamento de Alta Verapaz en la parte central de Guatemala, Wilson (1974) reporta la presencia de la Caliza Coban con espesor aproximado de $1344 \mathrm{~m}$, de los cuales los $300 \mathrm{~m}$ inferiores son dolomía, en la parte superior hay Nummoloculina heimi y rudistas probablemente del Albiano, según él, el contacto superior con la Formación Sepur es discordante y con un hiato del Cenomaniano al Santoniano. Leight y Buis (1979) consideran que en el subsuelo del área Rubelsanto, parte de la Formación Coban ' $\mathrm{B}$ ' y parte de la ' $\mathrm{C}$ ' fueron truncadas hasta la base de la ' $\mathrm{A}$ ', no se conoce el detalle petrológico de estas tres últimas unidades informales.

En los alrededores de la Ciudad de Guatemala hay afloramientos de rocas carbonatadas del Cretácico Inferior, a las cuales Wilson (1974) nos les da un nombre estratigráfico formal, solamente les llama 'Cemento Novella' y contienen miliólidos y macroforaminíferos, para el autor, el retrabajo de clastos de caliza del Albiano en esta localidad, indica que la erosión de los carbonatos de plataforma había comenzado. Al oriente de la Ciudad de Guatemala, aproximadamente a $110 \mathrm{~km}$ y cerca de la frontera con Honduras, Wilson (1974) describe la Caliza Buenavista, cuya edad varía del Neocomiano al Albiano, contiene caprinidos y se formó en un ambiente de plataforma somera, semejante al las rocas de Alta Verapaz y de la Ciudad de Guatemala; Wilson describe que en el Cenomaniano temprano hay un evento volcánico importante que produce flujos de lava, acompañados de conglomerados con clastos de lava y fragmentos de fósiles. También en el sureste de Guatemala (a $40 \mathrm{~km}$ del límite con Honduras) Burkart et al. (1973) detallan el Grupo Yojoa con cuatro formaciones, del cual la Formación Atima (localidad tipo en Honduras) es de una edad probable del Albiano Medio o Superior y contiene en su parte superior un conglomerado con clastos de caliza masiva y arenisca.

En Jocotan (15 km al oeste de la frontera con Honduras) la Formación Las Lajas, tiene en los sedimentos calcáreos de cuenca del Albiano Superior-Cenomaniano Inferior las evidencias de un vulcanismo contemporáneo, dadas por la presencia en la parte superior de la formación de una arenisca tobacea fina, al terminar el disturbio volcánico del Cenomaniano Inferior, retornaron las condiciones normales de sedimentación carbonatada (Wilson, 1974).

Blount y Moore (1969) aportan datos importantes sobre los litoclastos, pero no hay descripción de la geometría y posición relativa de estos, todo parece ser coincidente con un paleokarst, faltan detalles de campo. Wilson (1974), Leight y Buis (1979) describen en el subsuelo "formaciones truncadas" y Burkart et al. (1973) describen una 'discordancia' con conglomerados de clastos de lava y fragmentos de fósiles en las rocas superficiales. En el caso de los tres autores antes mencionados es indispensable realizar un detalle de campo en la petrología y fábricas de los conglomerados.

\section{Honduras}

En el oeste de Honduras (figuras 4 y 5) en una localidad llamada El Carrizal, la secuencia de rocas Yojoa-La Misión, consiste de caliza litográfica con macrofauna esparcida y algunos miliólidos muy grandes y está cubierta discordantemente por lutitas rojas yesiferas y lutitas tobaceas, así como areniscas y limolitas.

En el centro de Honduras, al noroeste de Tegucigalpa las rocas carbonatadas de plataforma del Cretácico Inferior y del Cretácico superior temprano, están separadas por una gran discontinuidad estratigráfica seguida de actividad volcánica y el depósito de material clástico grueso (Wilson, 1974), lo anterior se observa en las secuencias de rocas de Santa Barbara y Yojoa-La Misión. Hay una unidad litológica llamada Formación Esquias probablemente del Cenomaniano que separa a 
dos secuencias de capas rojas, unas superiores y otras inferiores, en la secuencia inferior hay clásticos de caliza del Albiano, y en la superior clásticos de rocas volcánicas. La actividad volcánica de corta duración que se inició en el Cenomaniano (figura 2), es el producto de los movimientos de deformación en la corteza, los cuales son notables en la 'América Central Nuclear' (Wilson, 1974).

Como en el caso de Guatemala, Wilson (1974) no presenta detalles de la "discontinuidad estratigráfica seguida de actividad volcánica y el depósito de material clástico grueso", pero podría tratarse de un evento paleokárstico importante que anteceda a la actividad volcánica mencionada, hay que ver evidencias de campo y detalles de la petrología.

\section{Magnitud de las causas para la formación de los paleokarsts y erosión subaérea}

En el este de Texas en una porción geológica, estructuralmente poco deformada Vierbuchen y Oestmann, (1987) calcularon por medio de estratigrafía sísmica, paleontología y geología de subsuelo, que durante el 'Cretácico medio' (Albiano Superior-Cenomaniano) hubo una caída del nivel del mar del orden de los 60 a 100 metros en la provincia geológica del Golfo de México.

Es importante resaltar que en México, no sólo hay el evento de erosión del Albiano Superior, además en algunas plataformas calcáreas hubo erosión de los sedimentos, o no depósito?, durante el Coniaciano-Santoniano (Sierra del Abra), o bien, desde el Cenomaniano al Oligoceno (Faja de Oro), entonces es importante distinguir estos eventos.

\section{Conclusiones y recomendaciones (SECC)}

1. Durante el Albiano Superior en México hubo un periodo de exposición subaérea en las plataformas calcáreas, causado por un descenso del nivel del mar, que dio lugar a la formación del paleokarst de Dengandho, el cual ha sido estudiado por medio de la metodología de estudios petrológicos, de petrofábricas y estratigrafía.

2. En las plataformas de Coahuila, Valles-San Luis Potosí, Faja de Oro, Córdoba, Artesa-Mundo Nuevo, Chiapas, Guatemala y Honduras no hay suficientes evidencias en la literatura, para poder confirmar la presencia de paleokarsts. Se recomienda detallar la información de campo, del subsuelo (núcleos de roca), sísmica y de detalle petrológico con la metodología citada aquí para el estudio de la brechas en palaokarsts.
3. Fuera de México, Guatemala y Honduras, hay la comprobación de la existencia de un paleokarst en la plataforma de San Marcos en rocas del Albiano Superior.

En el Golfo de México, por medio de la geofísica se ha determinado la existencia de discordancias en el Albiano Superior en el 'Jordan Knoll' y en el escarpe de la plataforma de Campeche, estas evidencias indirectas no son suficientes para la confirmación de Paleokarsts en ambas áreas.

A futuro en la exploración petrolera del Golfo de México, y en las expediciones oceanográficas se recomienda la obtención de núcleos de roca, para comprobar la existencia de un paleokarst y la discordancia asociada.

\section{Agradecimientos}

El Dr. J. Eduardo Aguayo C. Investigador de la Facultad de Ingeniería de la UNAM y especialista en el estudio de rocas carbonatadas, enriqueció en forma sustantiva este documento con su experiencia en el estudio de las rocas kársticas, así como con sus observaciones. La Dra. Elia Ramírez A. Investigadora del Instituto de Geología de la UNAM especialista en palinología, impulsó en forma sustancial la calidad del manuscrito. A tres revisores anónimos que con sus atinadas observaciones sobre una reestructuración del artículo, lo mejoraron y enriquecieron.

\section{Referencias}

Aguayo-C.J.E. Sedimentary Environments and Diagenesis of a Cretaceous Reef Complex, Eastern Mexico. Centro Cienc. del Mar y Limnología. Universidad Nalcional Autónoma de México, 5:83-140. 1978.

Aguayo-C.J.E. The Middle Cretaceous El Abra Limestone at its Type Locality (Facies, Diagenesis and Oil Emplacement), Eeast-Central México. Revista Mexicana de Ciencias Geológicas, 15:1-8. 1998.

Aguayo-C.J.E., Basañez M.A., Bello R., Pimienta M. y Sosa A. Tectonic Evolution and Carbonate Sedimentary Environments During the Mesozoic at Reforma-Jalpa Area, Southeast Mexico. On: Crevello P.D. y Harris P.M. edits. Deep-Water Carbonates a Core Workshop, SEPM Workshop, No. 6, New Orleans, pp. 249-265. 1985.

Blount D.N. y Moore C.H. Depositional and Non-Depositional Carbonate Breccias. Chiantla Quadrangle. Guatemala. Bull. Geol. Soc. America, 80:429-442. 1969.

Boese E. Geología de los alrededores de Orizaba con un perfil de la vertiente oriental de la Mesa central de México. Boletín del Inst. Geológico de México, (13). 1899. 
Boese E. Reseña acerca de la geología de Chiapas y Tabasco. Inst. Geol. de México, 20: 5-100. 1905.

Burckhardt. Etude synthétique sur le Mésozoïque mexicain. Mémoires de la Société Paléontologique Suisse, IL:280. 1930.

Burkart B., Clemons R.E. y Crane D.C. Mesozoic and Cenozoic Stratigraphy of Southeastern Guatemala. Bull. Amer. Assoc. Petroleum Geol., 57:63-73. 1973.

Carrasco-V.B. Litofacies de la Formación El Abra en la Plataforma de Actopan, Hgo. Revista del Instituto Mexicano del Petróleo, 3:526. 1971.

Carrasco-V.B. Paleokarst in the Marginal Cretaceous Rocks, Gulf of Mexico. On: Bartolini C., Buffler R.T., Blickwede J. edits. The Circum-Gulf of Mexico and the Caribbean: Hydrocarbon Habitats, Basin Formation, and Plate Tectonics, Amer. Assoc. Petr. Geologists Memoir 79, pp.169-183. 2003.

Carrasco-V.B., Morales-P.P., Cienfuegos E. y Lozano-S.R. Geoquímica de las rocas asociadas al paleokarst cretácico en la Plataforma de Actopan: Evolución paleohidrológica. Rev. Mex. Ciencias Geol., 21:382-396. 2004.

Carrillo-Martínez M. Hoja Zimapán 14Q-e(7), Carta Geológica de México, Serie de 1:100 000, Resumen de la Geología de la Hoja Zimapán, Estados de Hidalgo y Querétaro, 33 p. 1998 (2000).

Carrillo-Martínez M., Valencia I.J.J. y Vázquez M.E. Geology of the Southwestern Sierra Madre Oriental Fold-and-Thrust Belt, East-Central Mexico: A Review. On: The Western Gulf of Mexico Basin, AAPG Memoir 75, pp. 145-158. 2001.

Castro M.J.T., Schlaepfer C. y Matínez E. Estratigrafía y microfacies del Mesozoico de la Sierra Madre del Sur, Chiapas. Bol. Asoc. Mex. Geol. Petroleros, 27:1-95. 1975.

García H.C. Geological and Geohydrological Studies for Angostura Dam, Chiapas, México. Bull. Geol. Soc. America, 84:17331742. 1973

Colorado L.D. y Cruz R.R. Tipología de yacimientos paleokarsticos, generalidades, aplicación en algunos ejemplos mexicanos y consideraciones regionales, Consejo de Recursos Minerales VIII Seminario Interno sobre Exploración Geológico-Minera, p. 214-238. 1980.

Coogan A.H., Bebout D.G. y Maggio C. Depositional Environments and Geologic History of Golden Lane and Poza Rica Trend, an Alternative View. AAPG Bulletin, 56:1419-1447. 1972.

Eguiluz de A.S. Discordancia cenomaniana sobre la Plataforma de Coahuila. Bol. Asoc. Mex. Geol. Petroleros, 41:1-17. 1991.

Enos P., Minero C.J. y Aguayo J.E. Sedimentation and Diagenesis of Mid-Cretaceous Platform Margin East-Central Mexico with Accompanying Field Guide. Dallas Geological Society. 168 p. 1983.

Garza G.R. Modelo sedimentario del Albiano-Cenomaniano en la porción sureste de la plataforma de Coahuila. Asoc. Mex. Geol. Petroleros, 25:311-339. 1973.

Gutiérrez G.R. Bosquejo geológico del Estado de Chiapas. En: Libreto guía de la excursión C-15, XX Cong. Geol. Int., pp. 9-32. 1956.
Guzmán E.J. Reef.-Type Stratigraphic Traps in México, Proc. $7^{\text {th }}$ World Petrol. Congress, Vol. 2, pp. 461-470, London, Elsevier. 1967.

Hovorka S.D., Dutton,A.R., Ruppel S.C. y Yeh J.S. Edwards Aquifer Ground-Water Resources: Geologic Controls on Porosity Development in Platform Carbonates, South Texas. The University of Texas at Austin, Bureau of Economic Geology, Report of Investigations 238, 75 p. 1996.

Hovorka S.D., Mace R.E. y Collins E.W. Permeability Structure of the Edwards Aquifer, South Texas-implications for aquifer management. The University of Texas at Austin, Bureau of Economic Geology, Report of Investigations 250, 55p. 1998.

Kerans Ch. Depositional Systems and Karst Geology of the Ellenburger Group (Lower Ordovician), Subsurface West Texas. The University of Texas at Austin, Bureau of Economic Geology, Report of Investigations No. 193, 63 p. 1993.

Leight R.A., Buis O.J. y Hall K.H. Exploración petrolera en Guatemala. En: R. del Arenal (editor), III Cong. Latinoamericano de Geología, Memoria, Tomo I (acta final), p. 75. 1979.

Loucks R.G. Paleocave Carbonate Reservoirs: Origins, Burial-Depth Modifications, Spatial Complexity and Reservoir Implications. Amer. Assoc. of Petrol. Geologists Bull. 83:1795-1834. 1999.

Loucks R.G. and Mescher P.K. Paleocave Facies Classification and Associated Pore Types, AAPG Southwest Section Annual Meeting, Dallas, Texas, March 11-13, 18 p. 2001,

Lucia F.J., Kerans Ch., Vander-Stoep G.W. Characterization of a Karsted, High-Energy, Ramp Margin Carbonate Reservoir. Taylor-Link West San Andres Unit, Pecos County, Texas, Bureau of Economic Geology. The University of Texas, Report of Investigation 208, 46 p. 1992.

Martínez M., Serrano E., Bartolo C., Toriz J., Espinoza M. y Vazquez R. Mesozoic Plays of the Cordoba Platform, Veracruz Basin. AAPG, Annual Convention Abstracts, p. A126. 2001.

Minero Ch.J. Sedimentation and Diagenesis Along an Island-Sheltered Platform Margin, El Abra Formation, Cretaceous of Mexico. On: Paleokarst, James N.P. y Choquette P.W. edits. Springer-Verlag, pp. 385-405. 1988.

Quintus B.R.L. Estudio geológico de la zona fosforítica de San Francisco, Municipio, de Pacula, Estado de Hidalgo. Libro guía de la excursión geológica a la región de Zimapán y áreas circundantes, Estados de Hidalgo y Querétaro. Sociedad Geológica Mexicana. 1982. Pp. 21-27.

Roehl P.O. Preliminary Guidebook to Selective Features of the El Abra Limestone Outcrops in the Sierra Madre Oriental, Mexico. Union Research Center, Unior Oil Company of California, 51 figs., páginado. 1968.

Schlager W. Depositional Bias and Environmental Change-Important Factors in Sequence Stratigraphy. Sedimentary Geology, 70:109-130. 1991.

Schlager W. y Philip J. Cretaceous Carbonate Platforms. On: Ginsburg R.N. and Beaudin B. eds. Cretaceous Resources, Events 
and Rhythms, Background and Plans for Research, NATO ASI Series, v. 304, pp. 173-195. 1990.

Steele D.R. Physical Stratigraphy and Petrology of the Cretaceous Sierra Madre Limestone, Part 1. On: West-Central Chiapas, Contributions to the Stratigraphy of the Sierra Madre Limestone (Cretaceous) of Chiapas, UNAM, Inst. Geología, Boletín 102, pp. 1-101. 1985 (1986).

Vahrenkamp V.C., Franseen R.C.W.M., Grötsch J. y Muñoz P.J. Maracaibo Platform (Aptian-Albian, Northwestern Venezuela. On: Simo T.J., Scott R.W. y Masse J.P. eds. Cretaceous Carbonate Platforms, AAPG Memoir 56, pp. 25-33. 1993.

Varela-S., Williams M.R.C. y Aguilera G.L. Oil and Gas Exploration and Production in Fold and Thrust Belts. On: Second Joint AAPG/AMGP Hedberg Research Symposium, Veracruz, Mexico, Co-Conveners Bishop R.S., Martell-A.B. and SanchezM. R., 6 p. 1997.

Vierbuchen R.C. y Oestmann M.A. Mid-Cretaceous Eustatic Sea Level Fall. Magnitde and Timing in Gulf of Mexico. Abstracts AAPG Program Annual Convention (June 7-10, Los Angeles, California). 1987.

Viniegra-O.F. y Castillo-T.C. Golden Lane, Veracruz, Mexico. On: Halbouty, M. T. ed., Geology of Giant Pretroleum
Fields. Amer. Assoc. Petroleum Geol. Memoir 14, pp. 309325. 1970.

Waite L.E. Part 2, Biostratigraphy and Paleoenvironmental Analysis of the Sierra Madre Limestone (Cretaceous), Chiapas. On: West-Central Chiapas, Contributions to the Stratigraphy of the Sierra Madre Limestone (Cretaceous) of Chiapas, UNAM. Inst. Geología, Boletín 102, pp. 103-245. 1985 (1986).

Wilson H.H. Cretaceous Sedimentation and Orogeny in Nuclear Central America. Bull. Amer. Assoc. Petroleum Geol., 58:13481396. 1974.

Wilson J.L. Carbonate Facies in Geologic History. Springer-Verlag. 409 p. 30 plates. 1975.

Williams R.C.T. Geologic Controls on Reservoir Performance in Muspac and Catedral Fields, Southeastern Mexico. On: The Western Gulf of Mexico Basin, AAPG Memoir 75, pp. 443472. 2001.

Zavala M.J.M. Estudio geológico del proyecto hidroeléctrico Cañón del Sumidero, Río Grijalva, Estado de Chiapas. Bol. Asoc. Mex. Geol. Petroleros, 23:1-92. 1971.

\section{Semblanza del autor}

Baldomero E. Carrasco-Velázquez. Egresado de la licenciatura en la carrera de ingeniería geológica, por la Facultad de Ingeniería de la Universidad Nacional Autónoma de México, se tituló con mención honorífica en 1962. En la Universidad de Texas, en Austin, obtuvo el grado de Master of Sciences en 1968. En el Posgrado en Ciencias de la Tierra de la UNAM (2001-2005), obtuvo el doctorado en 2005 con la tesis: "Paleokarst en rocas cretácicas de la Plataforma de Actopan, Hgo." Es profesor en la División en Ciencias de la Tierra de la Facultad de Ingeniería (UNAM) desde 1965 a la fecha con las asignaturas de de paleontología estratigráfica, petrología de rocas sedimentarias y petrología. Laboró en Petróleos Mexicanos durante 5 años y en el Instituto Mexicano del Petróleo durante 33 años. 